PHYSICAL REVIEW D 75, 099903(E) (2007)

\title{
Erratum: Meson-meson interactions in a nonperturbative chiral approach [Phys. Rev. D 59, 074001 (1999)]
}

J. A. Oller, E. Oset, and J. R. Peláez

(Received 26 April 2007; published 29 May 2007)

DOI: 10.1103/PhysRevD.75.099903

PACS numbers: 12.39.Fe, 11.80.Et, 13.75.Lb, 14.40.-n, 99.10.Cd

In Appendix A.1, we presented the equation for $G(s)$ in the general case with different masses, $M_{1}$ and $M_{2}$. However, the correct Eq. (A1) should read:

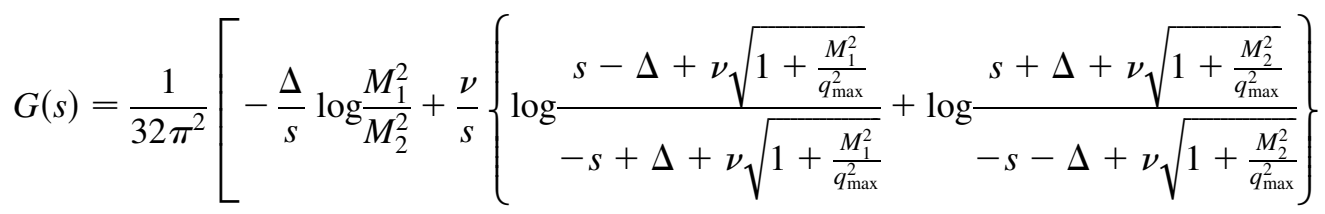

$$
\begin{aligned}
& \left.+2 \frac{\Delta}{s} \log \frac{1+\sqrt{1+\frac{M_{1}^{2}}{q_{\max }^{2}}}}{1+\sqrt{1+\frac{M_{2}^{2}}{q_{\max }^{2}}}}-2 \log \left[\left(1+\sqrt{1+\frac{M_{1}^{2}}{q_{\max }^{2}}}\right)\left(1+\sqrt{1+\frac{M_{2}^{2}}{q_{\max }^{2}}}\right)\right]+\log \frac{M_{1}^{2} M_{2}^{2}}{q_{\max }^{4}}\right] \text {. }
\end{aligned}
$$

In addition, $\Delta=M_{2}^{2}-M_{1}^{2}$, instead of $M_{1}^{2}-M_{2}^{2}$. The rest of the definitions are as before and the prescription for the logarithmic functions is such that $\operatorname{Im} G$ is given by Eq. (16).

The correct formula has been used in the calculations and the misprint of this equation has no repercussion on the results. 